\title{
Research on Fairness of IASB Conceptual Framework in Financial Reporting
}

\author{
Sun Yiting ${ }^{1, \mathrm{a}}$

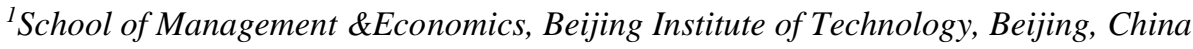 \\ asunheli3738@163.com
}

\begin{abstract}
IASB conceptual framework acts as a practical tool and assistance when developing IFRS. By probing into the degree to which IASB conceptual framework meets the given definition of fairness, it can be concluded that with an adequate recognition of users and their requirements, conceptual framework generally satisfies the fairness mentioned despite some flaws.
\end{abstract}

Keywords: IASB, Conceptual Framework, Fairness.

\section{财务报告中 IASB 框架的公平性探究}

\author{
孙伊婷 ${ }^{1, a}$
}

\footnotetext{
${ }^{1}$ 北京理工大学管理与经济学院, 北京, 中国

asunheli3738@163.com
}

摘要：国际会计准则理事会（IASB）的概念框架在制定国际财务报告准则时起到了实用工具和协助的作用。 通过探讨 IASB 的概念框架在多大程度上符合给定的公平定义，得出结论，即只要充分承认用户及其要求，概 念框架基本上满足上述公平性，尽管存在一些缺陷。

关键词：IASB，概念框架，公平

\section{1. 引言}

法律要求上市公司根据国际财务报告准则 （IFRS）定期编制财务报告。由于财务报告被广泛用 于为不同用户做财务决策, 因此确定 IASB 概念框架 作为实用工具和协助是否满足 “公平”一词是至关重 要的, 这是文章主要探讨的问题。

公平可以被解释为 “对相关公司的事务给予一个 整体平衡的观点, 没有实质性的差距” [1] 。决定 “公 平” 满意度的方式将满足用户的利益, 并要求视为一 个基本条件。值得注意的是, 财务会计使用者的利益 和需求不同, 导致了不公平的可能性。人们担心, 标 准设置偏爱编制者和专业会计师事务所, 而不是财务 报表的使用者 ${ }^{[2]}$ 。

文章框架将首先通过选取三个典型的财务报表 主要用户来讨论与他们理解公平性相关的用户各自 的需求和利益。在此之后, IASB 概念框架实现公平
性的方法将通过推理和列出具体的例子进行分析。然 而, 不能确保公平的限制性不可避免地存在, 这将在 三个主要方面加以阐明。最后, 根据讨论得出结论。

\section{2. 不同类型用户的需求}

从用户的角度来看, 对公平性的理解可以解释为 信息需求的满足。它们从明智的经济决策中获得的好 处取决于财务报告中信息的相关性和全面性 ${ }^{[3]}$ 。由于 对公平性的理解往往随着个人需求的不同而有所不 同，我们将通过确定报告用户各自的不同目标和信息 需求, 来了解对报告用户的看法, 主要是现有股东、 债权人和潜在投资者。现有股东是财务报告的主要用 户之一，其主要目标是实现财富最大化。为了实现其 目标, 需要通知现有股东公司的情况。具体来说, 他 们对公司控制的资产和负债的总额以及管理层如何 在经营活动中分配这些资源感兴趣。此外，与获得固 定利息的债权人不同，现有股东的利益是基于公司的 
业绩。因此，他们对经营的结果更为敏感，并需要一 个关于产生多少损益的准确和可靠的结果。

\section{1. 现有股东的需求}

现有的股东可能希望对其公司拥有全面的控制 权, 并做出后续的投资决策, 这样他们才能实现财富 的持续增长。此外, 他们需要关于其占总股本比例的 准确数据, 以便能够估计自己的股息份额, 以及他们 是否能够对公司拥有话语权。债权人需要财务报告才 能获得公司的真实情况, 并决定是否授信。资产、负 债和现金流等细节用于计算比率和评估公司按约定 偿还债务的能力。通过上述信息, 债权人可以确定该 业务的信用风险是否可控, 这影响了贷款合同的条 款。此外, 债权人还需要通过报表监测贷款的使用和 流动, 以防公司投资过度风险甚至非法项目。这两种 方法可以保护债权人免受损害由于过度贷款而造成 的经济损失。

\section{2. 债权人的需求}

债权人需要财务报告才能获得公司的真实情况， 并决定是否授信。债权人的主要目标是确保他们做出 正确的投资决策, 并实现未来的收益。因此, 为了实 现这些目标, 他们主要关注的是获取信息、分析财务 状况和做出预测。与现有股东一样, 潜在投资者主要 关注公司的盈利能力, 因此潜在投资者希望知道当前 获得的利润和支付的股息。更重要的是, 他们需要数 据来估计未来的现金流，这表明未来支付的股息和资 本利得 ${ }^{[4][5]}$ 。此外, 它们还需要提供信息, 以帮助它 们决定适当的投资金额、时间以及要投资何种类型的 股本。因此, 它们可能涉及到当前的股本数额、公司 的发展阶段, 以及公司对普通股和优先股的安排。

\section{3. 潜在投资者的需求}

潜在投资者的主要目标是确保他们做出正确的 投资决策, 并获得未来收益。因此, 为了实现这些目 标, 他们主要关心的是获取信息、分析财务状况和做 出预测。与现有股东一样, 潜在投资者主要关注公司 的盈利能力, 因此潜在投资者希望知道公司实现的当 前利润和支付的股息。更重要的是, 他们需要数据来 估计未来的现金流, 这表明未来支付股息和资本收益 ${ }^{[4][5]}$ 。此外, 他们亦需要资料, 以协助他们决定适当 的投资额、时间及投资于哪类股本。因此, 他们可能 会关注目前的股本金额, 公司的发展阶段, 以及公司 对普通股和优先股资本的安排。最后, 潜在投资者需 要可靠的潜在投资风险警报, 作出公正的决策, 防止 严重亏损 ${ }^{[6]}$ 。

\section{IASB 实现公平的路径}

在确定了主要用户的目的和需求后, 我们将研究 IASB 概念框架如何满足这些需求, 这是指实现所给
出的公平性。在框架的指导下, 国际财务报告准则有 助于提供财务报告和会计的真实报告。此过程为广大 用户提供符合决策一般要求的决策有用信息。因此, 下面三段将进一步探讨当前的框架和标准如何满足 用户的要求和公平感知。

\section{1. 提供一系列财务数据}

为了满足现有股东的需求, IASB 的概念框架需 要准备几份财务报表, 其中提供了一系列的财务数 据。财务状况报表反映了公司在报告日的资产、负债 和权益。特别是留存收益, 即再投资于公司的利润, 代表了现有股东的潜在收益 ${ }^{[7]}$ 。损益表和其他综合收 益表提供了关于该公司在上一财政年度的收入的直 接信息。通过分析，现有股东可以估计公司当前的盈 利能力、投资效率, 更重要的是, 实现了利润的增长 趋势。现金流量表主要显示现金的流动。最重要的是, 它可能是对破产和对继续经营的威胁的早期预警 ${ }^{[8]}$ 。 股权变动表显示了已发行的新股和已支付的股息。现 有股东可以了解其股权比例、非流动资产增值和宣布 的股息的变化。基本上, 上述财务报表在一定程度上 可以满足现有股东对信息的主要需求。

\section{2. 促进真实反映公司的财务状况}

审慎的定义和概念框架原则可以在一定程度上 满足债权人的要求, 从而促进公司财务状况的真实反 映。例如，在概念框架中对 “责任” 的定义是 “该实 体目前因过去而转移经济资源的一种义务” “事件” ${ }^{[9]}$ 。“经济资源”一词的使用澄清了, 责任不是经济 利益的最终流出, 而是不可避免的转移责任, 这减少 了关于股票期权等基于股票的支付的争议 ${ }^{[10]}$ 。此外, 概念框架最重要的原则之一是 “实质而不是形式”。 例如, 即使 “销售和回购” 的活动以法律形式发生, 交易也被收取 “责任” 而不是 “收入”, 因为其实质 内容是表外财务。这一原则为不同的公司和公司政策 提供了一个统一和明确的财务报告标准, 降低了操纵 财务报表的风险，从而保护了债权人的利益。

\section{3. 提供多种信息}

财务报表以公司控制的资源和收入为重点, 为潜 在投资者提供各种信息。因此，他们可以对公司的运 营有一个全面的了解。此外, 对于潜在的投资者来说, 使用财务报表中的特定数字进行数学计算来获得财 务指标, 然后评估公司的盈利能力、流动性、偿付能 力和持续经营也是有意义的。此外, IASB 的概念框 架强调了财务报表中几个定性特征的反映, 这可以有 效地帮助潜在投资者。例如， “可比性” 需要一个明 确的财务报表格式, 并防止一个公司不必要地和频繁 地改变其会计政策。它确保了财务报表之间的水平和 垂直比较都很有意义。这有助于潜在投资者判断一家 公司的发展趋势 ${ }^{[11]}$ 。德弗朗哥等人的公司特定的可比 性度量捕获了具有相似经济特征的公司具有可比性 
会计信息和类似财务报表信息的程度。使用 1981 年 至 2013 年的一个美国大样本，会发现，可比的财务 报表显著减少了公司的现金持有量。在经济意义方 面, 研究表明, 可比性的一个标准差增加与现金持有 量比均值减少有关, 这在经济上是非常显著的。为此 进一步证明，财务报表可比性对现金持有的影响是由 融资限制、财务报告质量和公司治理所调节的。这些 结果对替代回归规范和可比性和现金持有的替代代 理是稳健的 ${ }^{[1]}$ 。可比较性的问题出现在涉及许多部门 和分支机构的关于财务报表、其质量、信息作用等的 科学文献中。关于财务报表可比性的文献的情况有所 不同。这是金融会计研究的主要兴趣点之一, 因此关 于该课题的文献十分丰富。过去, 关于财务报表可比 性的研究主要采用财务报表输入的比较, 涉及不同国 家会计规则或会计系统中会计规则的相似性, 由此分 别分析不同公司之间的比较优势 ${ }^{[12]}$ 。

\section{IASB 的局限性}

正如上面所讨论的, IASB 的概念框架已经设置 了原则来确保财务会计和报告的公平性。它在大多数 情况下都很有效。然而, 在一些财务丑闻中, 除了贪 婪的公司管理人员外, 财务会计和报告系统都将受到 指责。其罪魁祸首是概念框架的一些局限性。回顾了 其他学者的论文、2018 年版本的 IASB 概念框架以及 丑闻案例, 总结为三个主要限制。

\section{1. 未能反映经济的现实情况}

在概念框架的指导下, 财务报告在某种程度上不 能以现实的方式反映经济现实。根据麦金塔什 ${ }^{[13][14]}$, 当经理在财务报告和组织交易中使用判断来改变财 务报告, 误导一些利益相关者了解公司的潜在经济业 绩或影响时, 就会发生收益管理 ${ }^{[13]}$ 。上述失败的内在 原因是, 报告中的会计语言是构建真理的工具, 而不 是透明和呈现真理。在某些情况下, 会计师修正了他 们的工作, 以满足国际财务报告准则的某些标准, 这 可能会扭曲原始数据的准确性和真实性。希望将出现 一个概念框架, 所有的会计实践、知识、规则和原则 都可以建立在其基础上, 从而保证会计报表将公平地 呈现 (或对企业的经济金融交易和相关事件提供一个 真实和公平的看法）。对该行业在这个方向上的努力 的简要历史描述, 将作为一些使这一思路产生问题的 后结构主义立场的背景 ${ }^{[13]}$ 。会计概念和原则声称, 真 实收益是指当期企业真实财务财富正式准则的增加, 即对投资者、债权人和其他利益相关者的决策有效性 是财务报告的基本目标, 相关性和可靠性被认为是主 要的定性特征 ${ }^{[14]}$ 。另一个原因是, 会计报告中的数字 是可操作的。例如, 当在分类账和账目中精心分配要 素编制财务报告时, 将进行避税。这种真理的构建会 损害陈述的公平性，从而给用户呈现错误的信息。许 多此类会计措施是直接从财务报表中采取的, 有些是 基于对财务报表数字进行的简单算术运算, 例如共同 定额, 还有一些是相当复杂的计算, 包括从会计数字
回归中得出的数字。在许多情况下, 会计或会计派生 数字代表因变量, 也就是说, 被解释的现象是通过会 计操作。因此, 从事严谨研究的会计学者表现得好像 这些会计数字对于以 “严谨” 的方式分析和评价会计 现象特别有用 ${ }^{[15]}$ 。相当具有讽刺意味的是, 当前的会 计决策基于与严格的会计研究相同的假设, 导致了会 计数据对严格的会计财务研究的有用性并不是特别 合理 ${ }^{[15]}$ 。

\section{2. 未能形成会计系统}

概念框架并没有塑造正确捕获和反映业务风险 的会计系统, 这可能会影响用户的公平感知。概念框 架内披露条例不足, 导致非财务信息披露不足甚至不 披露, 特别是在中小企业中。最重要的是, 专业判断 还需要决定要披露的内容。这两个因素将通过减少信 息披露, 共同促进业务风险的隐藏。例如, 公司可能 会隐藏不利的非财务信息, 导致高估其偿还债务的能 力。因此，债权人的贷款可能远远超过公司的承受能 力。回顾起来，许多敢于冒险的债权人并没有因为披 露警告不足而躲避商业巨头的崩溃。

\section{3. 公平的不确定性}

在各种组织中不加选择地应用 IASB 概念框架并 不能确保公平。非营利组织 (NFP) 组织在世界经济学 中发挥的作用越来越重要，因为政府将其服务外包给 NFP 部门 ${ }^{[16]}$ 。鉴于非盈利 (NFP) 组织的规模不断扩 大，他们对金融活动的报告引起了更大的关注。由于 NFP 部门的会计目的与盈利性部门差异很大, 主要针 对营利部门定制的概念框架和标准可能无法满足 NFP 部门的财务需求 ${ }^{[17]}$ 。根据 Ralph（2020）的问卷, NFP 的首席执行官选择了两个关于是否应该使用单一的 概念框架的首选选项, 可以作为一套兼容两个部门的 单一标准得出结论, 但通过额外的段落或标准解决特 定部门的问题。这是概念框架的另一个差距, 以确保 公平。

\section{5. 结论}

通过从现有框架中识别主要用户的实际需求和 支持性术语, 批判性地讨论了 IASB 概念框架在多大 程度上满足给定的公平定义。基于以上讨论, 可以得 出结论: 随着对用户及其需求的充分认识, 概念框架 虽然存在一些缺陷, 但一般都满足所提到的公平性。 事实上, 在世界范围内, 人们都普遍接受现有的框架。 在过去的几十年里, 人们进行了不断的修改和改进, 以便更好地支持不同的群体实现他们的目标。总之, IASB 的概念框架仍有发展的空间。

\section{REFERENCES}

[1] Harris, N. (1987) Fairness in Financial Reporting. Journal of Applied Philosophy, 4(1):77-88. 
[2] Alnoor, B., David, B. \& Prabhu, S. （2019） Does greater user representation lead to more user focused standards? An empirical investigation of IASB'S approach to standard setting. J. Accounting Public Policy, 38:65-88.

[3] Baksaas, K. \& Stenheim, T. (2019) Proposal for improved financial statements under IFRS. Cogent Business \& Management, 6.

[4] Elkhashen, E. M. \& Ntim, C. G. (2018) Accounting and philosophy: The construction of social reality framework. Journal of Accounting and Taxation, 10(3):29-36.

[5] Elliott, B. \& Elliott, J. (2009) Financial Accounting and Reporting. 13th ed. Edinburgh: Financial Times Prentice Hall.

[6] Yoon, B., Roh, T., Jang, Y. J. \& Yun, D. (2019) Developing a Risk Signal Detection System Based on Opinion Mining for Financial Decision Support. Sustainability, 11(16):1-26.

[7] Arnott, R.D. \& Asness, C. S. (2003) Surprise! Higher dividends $=$ higher earnings growth. Financial Analysts Journal, 59 (1):70.

[8] Laitinen, E. K. （1992） Prediction Of Failure of A Newly Founded Firm. Journal of Business Venturing, 7 (4):323-340.

[9] IASB Board. (2008) Conceptual Framework Phase B - Elements and Recognition. https:// www. iasplus. com/en/meeting-notes/iasb/2008/agenda_0806/age nda 997 .

[10] IFRS. (2004) IFRS 2 Share-based Payment. https://www.iasplus.com/en/standards/ifrs/ifrs2\#. (Accessed 3 Dec 2020)

[11] Habib, A., Hasan, M.M. \& Al-Hadi, A. (2017) Financial statement comparability and corporate cash holdings. Journal of Contemporary Accounting \&Economics, 13(2):304- 321.

[12] Czerny, M., Firkowska-Jakobsze, Z. \& Hońko, S. (2019) A Comparability of Information in the Financial Statements of Gaming Companies. Wydawnictwo Uniwersytetu Ekonomicznego We Wrocławiu, 24 (4):27-44.

[13] Macintosh, N.B. (2006) Accounting truth, lies, or "bullshit"? A philosophical investigation. Accounting and the Public Interest, 6(1):22-36.

[14] Macintosh, N.B. (2009) Accounting and the truth of earnings reports: philosophical considerations. European Accounting Review, 18(1):141-175.
[15] Williams, P.F. (2014) The myth of rigorous accounting research. Accounting Horizon, 28(4):869-887.

[16] Connolly, C., Hyndman, N. \& McConville, D. (2013) UK charity accounting: an exercise in widening stakeholder engagement. The British Accounting Review, 45:58-69.

[17] Van Staden, C. \& Heslop, J. (2009) Implications of applying a private sector based reporting model to not-for-profit entities: the treatment of charitable distributions by charities in New Zealand. Australian Accounting Review, 19:42-53. 